\title{
Emerging treatment options for the mucopolysaccharidoses
}

This article was published in the following Dove Press journal:

Research and Reports in Endocrine Disorders

5 November 2012

Number of times this article has been viewed

\author{
Roberto Giugliani ${ }^{1-6}$ \\ Andressa Federhen 1,4 \\ André Anjos Silva ${ }^{1,5}$ \\ Camila Matzenbacher \\ Bittar ${ }^{1,5}$ \\ Carolina Fischinger Moura \\ de Souza' \\ Cristina Brinckmann \\ Oliveira Netto' \\ Fabiana Quoos Mayer ${ }^{2,7}$ \\ Guilherme Baldo 1,2,8 \\ Ursula Matte ${ }^{1-5}$ \\ 'Medical Genetics Service, ${ }^{2}$ Gene \\ Therapy Center, Hospital de \\ Clínicas (HCPA), Porto Alegre, RS, \\ Brazil; ${ }^{3}$ Department of Genetics, \\ ${ }^{4}$ Postgraduate Program in Child and \\ Adolescent Health, ${ }^{5}$ Postgraduate \\ Program in Genetics and Molecular \\ Biology, Universidade Federal do \\ Rio Grande do Sul (UFRGS), Porto \\ Alegre, RS, Brazil; ${ }^{6}$ National Institute \\ of Population Medical Genetics \\ (INAGEMP), Porto Alegre, RS, Brazil; \\ ${ }^{7}$ Fundação Estadual de Pesquisa \\ Agropecuária (FEPAGRO), Eldorado \\ do Sul, RS, Brazil; ${ }^{8}$ Department of \\ Biophysics, Universidade Federal do \\ Rio Grande do Sul (UFRGS), Porto \\ Alegre, RS, Brazil
}

Correspondence: Roberto Giugliani Medical Genetics Service, Hospital de Clínicas de Porto Alegre, Rua Ramiro Barcelos 2350, 90035-903,

Porto Alegre, RS, Brazil

Tel +55 5l 33596338

Fax +55 5I33598010

Email rgiugliani@hcpa.ufrgs.br
Abstract: The mucopolysaccharidoses (MPS) are a group of diseases arising from one of eleven different enzyme defects, each one affecting one single step of the degradation pathway of glycosamynoglycans. Several developments in the understanding of the MPS have occurred since the first clinical report about their occurrence in 1917: the nature of the storage product was recognized, a useful biomarker (mucopolysacchariduria) was developed, the enzyme defects became identified, and the gene defects were elucidated. The first successful treatment for MPS diseases was bone marrow transplantation, which was introduced for the therapy in 1980. Over the last decade, a whole new set of therapeutic approaches have become available or are currently in development to address MPS. Intravenous enzyme replacement therapy, already approved for MPS I, II, and VI, will possibly be available for MPS IVA and for MPS VII within the next few years. Intrathecal enzyme replacement therapy (tested in animals and already reported in a few patients) may become a tool to treat or prevent the central nervous system (CNS) manifestations which occur in several MPS. Substrate inhibition therapy using small molecules which cross the blood-brain barrier is also being tested for MPS types with CNS manifestations. In vitro studies point out that chaperones may also be of therapeutic value when the main cause is protein misfolding and retention at the endoplasmic reticulum. Stopcodon read-through strategy has been tried in preclinical studies with MPS models caused by nonsense mutations. Preclinical studies assessing gene therapy also show quite encouraging results, and this modality of treatment is now moving toward clinical development. The use of neural stem cells in MPS types which have CNS involvement is also promising. It is important to point out that MPS diseases are hard to treat, and demand specific therapies with a broad range of supportive measures. An additional challenge arises from the high cost of these technology intensive approaches, and this needs to be addressed if the treatments are to become widely available. Finally, since there is evidence that early diagnosis followed by early treatment considerably improves the health outcomes for these patients, newborn screening is becoming increasingly important for the early diagnosis of affected patients.

Keywords: mucopolysaccharidoses, glycosaminoglycans, treatment, enzyme replacement therapy, gene therapy, lysosomal diseases

\section{Introduction}

The group of the mucopolysaccharidoses (MPS) includes diseases arising from eleven different enzyme defects, each one affecting one single step in the degradation pathway of glycosaminoglycans (GAGs). In this step, particular species of GAGs are accumulated, and a characteristic picture is observed in the patients who present with one of the eleven MPS types. In each MPS type, a broad heterogeneity of manifestations is usually observed, tentatively related to residual enzyme activity, 
which could be conditioned by the underlying gene defect (with a trend to be more severe when gene rearrangements or frameshift/nonsense mutations occur, and more attenuated when missense mutations are involved). Many developments in understanding the MPS have occurred since they were first reported in 1917: the nature of the storage products was recognized, a useful biomarker (mucopolysacchariduria) was developed, the enzyme defects became identified, and the gene defects in all the diseases became known. ${ }^{1}$

The first successful treatment for these diseases was bone marrow transplantation (BMT), introduced in 1980. BMT or hematopoietic stem cell transplantation (HSCT) is still used, and its main indication at present is for therapy of the severe form of MPS I (Hurler disease) when the transplantation can be performed before the age of 2.5 years or when the patient presents with an intelligence quotient greater than 70. If performed later, BMT/HSCT will not prevent the cognitive decline that occurs in this form of the disease, and other forms of therapy will be preferred due to the high mortality and morbidity rates associated with the transplantation procedure. For this reason BMT/HCST is not usually indicated for the more attenuated forms of MPS I or MPS VI, despite the reports of successful outcomes in these MPS types. BMT/ HSCT does not seem to be an option for MPS III and MPS IV, and its indication in MPS II is still controversial.

Following the success of enzyme replacement therapy (ERT) for the treatment of Gaucher disease, this method of treatment was also developed for MPS and is now available for MPS I (since 2003), MPS VI (since 2005), and MPS II (since 2006), and has certainly improved the outcome of patients affected by these MPS types.

The storage of GAGs still seems to be the primary problem in MPS, but it is now well known that this launches a cascade of pathogenic mechanisms, opening the door for the use of different therapeutic approaches targeting different disease mechanisms.

This review does not focus on the available BMT/ HSCT and ERT approaches, but rather concentrates on the emerging treatment options for these diseases, including intravenous ERT for further MPS types, intrathecal ERT, substrate reduction therapy, the use of chaperones, stopcodon read-through (SCRT), gene therapy, and the use of neural stem cells.

\section{Intravenous enzyme replacement therapy}

ERT involves regular-infusions of a recombinant product which is similar to the deficient enzyme missing in the specific MPS type. Before the advent of therapies targeting the deficient enzyme activity, the treatment of MPS was mainly focused on the prevention and care of complications, which is still a very important aspect in the management of these patients. ${ }^{2,3}$ ERT is the main specific treatment already available for some types of MPS, which brings substantial benefits to the patients. Over the last few years, the prognosis of the disease in MPS patients has improved considerably due to better understanding of the complications, the better supportive care provided, and also due to the specific therapies developed for some MPS types. ${ }^{4}$ However, improvements are more noticeable in some areas (visceromegaly, respiratory system, heart muscle, joint mobility, endurance) than in others (heart valves, cornea, skeletal system, and central nervous system [CNS] manifestations). ${ }^{5-7}$

It is important to mention that the production of antibodies against the recombinant enzymes may impact the efficacy of the enzyme replacement therapy, and some research initiatives are in progress to develop protocols to minimize this problem. ${ }^{8}$ In addition, the use of ERT before and immediately after HSCT has been proposed, as this would improve the patient's condition for the HSCT procedure, and would provide enzyme supply while the donor cells are still establishing themselves in the receptor (this would be even more important if the donor is a carrier). ${ }^{9}$

As clinical trials using ERT are already underway for MPS IVA, and possibly for MPS VII in the near future, we will focus on these conditions in this section. MPS IVA, also known as Morquio syndrome type $\mathrm{A}$, is a rare, autosomal recessive disorder caused by a deficiency of the lysosomal enzyme N-acetylgalatosamine-6-sulfate-sulfatase, which catalyzes one of the steps of the catabolism of the GAGs keratan sulfate and chondroitin-6-sulfate. ${ }^{10}$ The multisystemic impact of this storage results in skeletal dysplasia, decreased endurance, and impaired quality of life. Generally, MPS IVA patients with a severe form of the disease do not survive beyond 20 to 40 years, whereas those patients with an attenuated form may survive longer. Mortality is commonly due to cardiorespiratory or neurological complications. ${ }^{1,11}$ As no specific therapy for MPS IVA has been available until now, palliative care has been the primary treatment modality.

ERT is now being developed for MPS IVA, and preliminary data from preclinical studies ${ }^{12,13}$ and from Phase I/II clinical trials indicate the sharp decrease of urinary keratan sulfate excretion after a few weeks of treatment, with an additional improvement in a 6-minute walking test and a 3-minute stair climb test; these results are similar to those observed in clinical trials of ERTs for MPS I, II, and VI. 
Adverse reactions are also comparable to those observed with the use of ERT across other MPS. ${ }^{14}$ However, more conclusive data on the efficacy of this therapy will not be available until the results of the Phase III clinical trial are reported, and these are to be announced in early 2013. It may be anticipated that as the skeletal system is prominently affected in this type of MPS, early treatment would be important to reduce the burden of these manifestations, as bone is a difficult tissue to treat with ERT.

MPS VII (also known as Sly syndrome) results from a deficiency of $\beta$-glucuronidase (GUSB) and is inherited as an autosomal recessive trait. Affected patients share many clinical features with patients with other forms of MPS, including shortened life span, mental retardation, organomegaly, and the bone and joint abnormalities that are collectively referred to as dysostosis multiplex. ${ }^{15}$ Comprehensive studies of ERT in animal models of MPS VII (especially with mice, dogs, and cats), which have been playing an important role in the development of ERT for related disorders (including other lysosomal storage disorders [LSDs]), ${ }^{16,17}$ are promising. There is hope that a clinical development program of ERT for MPS VII will be starting shortly, as an Orphan Drug Designation was already granted for this product. The disease has a broad spectrum of clinical presentations, from hydrops fetalis to milder forms with normal intelligence, and it would be expected that ERT would provide greater benefits in the more attenuated forms, as the blood-brain barrier (BBB) may prevent enzyme therapy from directly helping the brain, as is the case with ERT in the other MPS which have involvement of the CNS. ${ }^{18}$

\section{Intrathecal enzyme replacement therapy}

It is known that some types of MPS develop CNS manifestations, which can include cerebral tissue abnormalities (leading to progressive cognitive deficit), and/or spinal cord compression (leading to myelopathy), and/or obstruction of cerebrospinal fluid reabsorption (leading to high-pressure communicating hydrocephalus). As in other affected areas (bone, joints, heart, airways, eye, etc), a reduction of GAGs in the CNS is imperative in order to revert, stop, or at least slow down the progression of the devastating effects of the GAGs accumulation in the brain and meninges.

As the $\mathrm{BBB}$ prevents the enzyme from reaching the brain in significant quantities, the intravenous ERT does not address the neurological complications of the disease. Recent animal studies suggest that intravenously administered enzymes can cross the BBB in small amounts; however, there is no conclusive evidence that this also occurs in humans. The attempt to deliver the recombinant enzyme intrathecally has been shown to enhance the enzyme activity levels in the brain (three- to 20-fold heterozygote levels). ${ }^{19}$

The intrathecal administration of recombinant arylsulphatase B resulted in a reduction in GAGs storage in the dura of MPS VI cats. ${ }^{20}$ Thus, intrathecal ERT is an effective way to introduce enzymes into the CNS in animal models and, although there is still no evidence that shows that it may prevent cognitive impairment in MPS I, some studies have demonstrated behavioral improvement in animal models of other LSDs when enzymes are provided directly into the brain. ${ }^{19}$ Other studies have shown that intrathecal ERT administered every 3 months adds substantial benefit to high-dose intravenous ERT for the treatment of CNS storage in MPS I in the canine model. The primary disadvantage of direct CNS delivery is the invasive nature of the procedure. ${ }^{21}$ Furthermore, we should mention that, with intratechal ERT, the distribution of enzymes within the brain may be imperfect, especially in larger brains, and the benefits of intrathecal ERT for subjects with established CNS manifestations may be difficult to achieve.

Two case reports have described successful delivery of recombinant enzymes intrathecally in adult patients with MPS I and MPS VI with spinal cord compression. In studies of canine models, the MPS I patient received four injections of $3 \mathrm{~mL}$ of laronidase (approximately $1.74 \mathrm{mg}$ of enzyme) at monthly intervals. ${ }^{22}$ The patient had improved gait, respiratory parameters, and decreased use of pain medications, without adverse events. ${ }^{23}$ The MPS VI patient had been presenting with a slow, but apparently steady improvement in sensitivity to hot and cold and reflexes; however, walking capacity decreased progressively. It was concluded that in situations of high surgical risk or less advanced bone disease, the intrathecal ERT approach may be considered as therapeutic or adjunct therapy for the MPS types for which ERT is available. ${ }^{24}$

The encouraging results which came from preclinical studies and case reports have paved the way for clinical trials investigating intrathecal therapy for MPS I (whether associated or not to BMT/HSCT), MPS II, and MPS IIIA, and these studies are currently in progress.

\section{Substrate reduction therapy}

Since intravenous ERT appears to be inefficient in treating the CNS manifestations of the MPS, studies on alternative therapeutic methods are being conducted. Substrate reduction therapy (SRT) has recently been shown to be a promising 
strategy for treatment of neuronopathic forms of MPS and other LSDs. ${ }^{25}$ The SRT aims to decrease the amount of stored material through the administration (usually oral) of small molecules that are capable of inhibiting the synthesis of the substrate stored in that specific disease, resulting in the restoration of the balance between synthesis and degradation. For the success of this type of therapy it is necessary that the enzyme has some residual activity, in order to turn over the decreased amount of stored substrate. In MPS disorders, the strategy for SRT would be the reduction of the formation of GAGs by inhibiting the enzymes needed for the synthesis of these molecules. ${ }^{26}$ Inhibiting the formation of the primary storage products should delay the development of pathologies in MPS disorders. The most important therapeutic strategy is based on the use of relatively small molecules that may cross the $\mathrm{BBB}$ and reach the $\mathrm{CNS}$, making SRT a potential strategy with which to approach the neurological problems associated with the MPS disorders. There are a number of inhibitors of GAGs synthesis, and we will comment briefly on the two which are considered the most important SRT agents for MPS.

\section{Genistein and other natural flavonoids}

The compound 5,7-dihydroxy-3-(4-hydroxyphenyl)-4H1-benzopiran-4-one, known also as genistein or 4,5,7trihydroxyisoflavone, has been demonstrated to be an inhibitor of GAGs synthesis in fibroblasts of patients suffering from various types of MPS. ${ }^{27}$ Subsequent studies on MPS II (Hunter syndrome) and MPS III (Sanfilippo syndrome) - the MPS types in which the neurological symptoms are severe - indicated that genistein, a natural isoflavone, can be effective in the treatment of some symptoms, including behavior and cognitive disabilities, in both mouse models (mice suffering from MPS II and MPS IIIB) and human patients. ${ }^{28-31}$ Improvement was noticed in the short-term treatment of animals with MPS II, and a complete correction of behavior was observed in long-term treatment of animals with MPS IIIB. ${ }^{29-30}$

In pilot clinical studies conducted for 1 year, it was demonstrated that treatment with a $5 \mathrm{mg} / \mathrm{kg} /$ day Genistein-rich isoflavone extract in 10 pediatric patients (3 to 14 years) suffering from MPS IIIA and MPS IIIB resulted in a statistically significant reduction in the concentration of urinary GAGs, changes in hair morphology, and improved cognitive function as assessed by special psychological tests. ${ }^{31}$ No adverse events related to the treatment were reported. The effects of genistein on neurological parameters in MPS animals and humans were assumed to be due to the ability of this isoflavone to cross the $\mathrm{BBB}$, which was previously demonstrated. ${ }^{32}$ The mechanism of genistein-mediated substrate reduction was proposed to be due to inhibition of tyrosine-specific protein kinase activity of the epidermal growth factor receptor. ${ }^{33}$

Recently, Kloska et $\mathrm{al}^{34}$ tested the effects of other flavonoids (apigenin, daidzein, kaempferol, and naringenin) on GAGs synthesis in cultured fibroblasts from MPS IIIA and MPS IIIB patients. They found that daidzein and kaempferol inhibited GAGs synthesis significantly and as efficiently as genistein. The study also showed that the combination of various flavonoids is more effective in inhibiting GAGs synthesis than the use of any of these compounds alone. Moreover, these compounds were able to reduce lysosomal storage in MPS IIIA fibroblasts and led to inhibition of GAGs synthesis by a mechanism other than blocking of the tyrosine kinase of epidermal growth factor receptors, as happens with genistein. This is important given that the use of tyrosine kinase inhibitors are associated with toxic and/or adverse effects like diarrhea and nausea, as well as skin and cardiac toxicity; therefore, their use in the treatment of MPS patients may result in compliance problems in the long term.

It should be mentioned that most of the trials with genistein are open-label, and the only controlled trial did not find an improvement in CNS function. ${ }^{35}$ There are suggestions that doses as high as $160 / \mathrm{mg} / \mathrm{kg}$ may be needed. ${ }^{36}$ The use of $150 / \mathrm{mg} / \mathrm{kg}$ for 12 months did not show side effects, but two out of eight patients showed deterioration during this period. ${ }^{37}$ In conclusion, although natural flavonoids may be an option for SRT in the MPS, further clinical trials are needed to evaluate the efficacy and safety of this potential treatment, and to establish recommended doses.

\section{Rhodamine B}

Rhodamine B is a coloring agent also known as DMC Red 37 that used to be used in cosmetics such as lipsticks and soaps. In vitro experiments in cultured human lip fibroblasts showed that rhodamine $B$ at concentrations of $25 \mu \mathrm{g} / \mathrm{mL}$ and $50 \mu \mathrm{g} / \mathrm{mL}$ led to a reduction in GAGs synthesis. ${ }^{38}$ Roberts et $\mathrm{al}^{39,40}$ demonstrated that rhodamine $\mathrm{B}$ is an effective inhibitor of GAGs production in vitro and in vivo in the MPS IIIA mouse model; however, the toxicity profile of rhodamine $B$ appears to be unacceptable for its use as a drug for humans. Although recent studies suggest that long-term treatment with low doses of rhodamine B is safe in mice, ${ }^{41}$ it is still not clear whether its safety profile would be the same for humans, and whether treatment with such low doses may be effective in improving MPS symptoms, or at least stopping or slowing down disease progression. It is important to mention that 
although rhodamine B may decrease the GAGs storage, its use possibly would not affect the levels of gangliosides and other neuronally-stored materials, which seem to contribute to the pathophysiology of MPS disorders.

\section{Pharmacological chaperones}

Lysosomal enzymes are synthesized and secreted into the endoplasmic reticulum (ER) in a largely unfolded state. An efficient intracellular system ensures that only properly folded proteins are transported to the Golgi apparatus for further maturation. Misfolded enzymes are rapidly degraded by the proteosome. Specific molecules, called "pharmacological chaperones," are able to increase residual enzyme activity by rescuing misfolded mutant proteins from rapid endoplasmic reticulum-associated degradation, and promoting the processing and trafficking of mutant enzymes to the lysosomes. As a result, the enzyme is still able to maintain its function, despite the initial misfolding caused by a missense mutation. ${ }^{42}$

Pharmacological chaperones (PC) therapy has been proposed and investigated as a potential treatment for many genetic diseases, including LSDs, that result from misfolded and/or unstable proteins. ${ }^{43,44}$ As already pointed out, the CNS manifestations of the LSDs do not respond to intravenous ERT due to the inability of these exogenous enzymes to cross the BBB. Other problems include insufficient delivery to relevant tissues and organs, immunogenic reactions, and tolerability. ${ }^{45,46}$ The PC, on the other hand, are small molecules that could be administered orally and could cross the BBB. Despite being a novel approach, there are a few drugs that have been proposed as PC, which are already being investigated in clinical trials. ${ }^{44}$ The treatment of metabolic diseases with competitive inhibitors such as chemical chaperones at subinhibitory intracellular concentrations was first proposed by Fan et al. ${ }^{47}$ They presented evidence that administration of deoxygalactonojirimycin at low concentrations effectively enhanced mutant lysosomal alpha-galactosidase A activities in lymphoblasts from Fabry patients with R301Q or Q279E mutations. Since then, PC have been exploited for other lysosomal storage disorders such as Gaucher, Pompe, Tay-Sachs, Sandhoff, GM1 gangliosidosis, and Niemann-Pick C, as well as for the stabilization of a variety of nonlysosomal proteins of medical interest. ${ }^{2,48,49}$

One of the disadvantages of a chemical chaperone, however, is the fact that its treatment effect is restricted to patients with missense mutations. PC might not be able to rescue processing mutants where the mutation has affected the active site or where the folding defect is too great to be reversed. The compound must be hydrophobic or be able to enter the cells through an alternative pathway and reach the ER at concentrations sufficient to promote folding. The concentrations of pharmacological chaperone required for rescue might be too high and produce negative side effects. As in Pompe patients, it may be assumed that only 10\%-15\% of these patients are amenable to enhancement therapy. ${ }^{2,50-51}$ In MPS, a few molecules have been studied for potential use as chaperones in MPS III. This group includes 2-acetamido1,2-dideoxynojirimycin and 6-acetamido-6-deoxycastanospermine, which may inhibit the involved enzyme NAGLU (deficient in MPS IIIB) at high concentrations, but these molecules may act as chaperones at lower concentrations. ${ }^{52}$ Feldhammer et $\mathrm{al}^{53}$ observed that glucosamine partially rescued the lysosomal membrane enzyme-deficient in MPS IIIC, which in most cases is retained in the ER and is not targeted to the lysosome. Suzuki et $\mathrm{al}^{54}$ are working on a new substance in $\beta$-galactosidase (deficient in MPS IVB) gene mutations, with some interesting preliminary results.

\section{SCRT}

SCRT is an approach in which there is suppression of premature termination codons (PTC) by the addition of a random amino acid in the PTC position, allowing for full protein translation. The translation termination mechanism is highly conserved among most organisms and is marked by three codons: UAG, UAA, and UGA. The nucleotide sequence around the stop codon also plays an important role in translation termination efficiency. Aminoglycosides, the main drugs used for SCRT purposes, appear to reduce the translation fidelity through proofreading inhibition, as they tend to increase the frequency of amino acid insertions in PTC sites and allow the ribosomes to continue translation. ${ }^{55}$ However, aminoglycosides have minimal effects on normal translation, as their termination signals in eukaryotes are surrounded upstream and downstream by sequences that increase translation termination efficiency. ${ }^{56}$ Therefore, the use of drugs as PTC suppressors in diseases such as the MPS, in which nonsense mutations form a significant portion of disease causing mutations, represents an alternative therapeutic approach.

A few studies have shown that SCRT is influenced by different factors. Keeling and Bedwell ${ }^{57}$ showed that efficiency of stop-codon suppression by gentamicin and amikacin was different for different codons and the adjacent nucleotides. A study of MPS I evaluated gentamicin as treatment for the IDUA p.Q70X and p.W402X mutations in CHO-K1 cells, and showed that the level of SCRT after gentamicin treatment was higher for PTC with lower fidelity stop-codons. ${ }^{58}$ 
Moreover, SCRT may not be effective in cases with markedly low levels of nonsense transcripts that occur due to the nonsense-mediated mRNA decay mechanism, which is responsible for the degradation of mRNA containing PTC, and was shown to be present in MPS. ${ }^{59,60}$ Linde et $\mathrm{al}^{61}$ showed that in cases of efficient nonsense-mediated mRNA decay, nonsense transcripts are markedly reduced, being insufficient in generating enough functional proteins even when gentamicin is provided.

Despite the influence of different factors, SCRT has been tested successfully for MPS I, which presents at least 15 PTC mutations. The first study that addressed the use of SCRT for MPS I showed that gentamicin treatment of fibroblasts heterozygous for the p.Q70X and p.W402X mutations restored alpha-1-iduronidase (IDUA) activity to $2.8 \%$ of normal levels. Moreover, the treatment was able to reduce the intracellular content of GAGs, resulting in a reduction in lysosomal vacuolation. ${ }^{62}$ Recently, Wang et al investigated the efficiency of SCRT to suppress the p.W392X nonsense mutation using gentamicin, G418, amikacin, paromomycin, and the designer aminoglycosides NB54 and NB84: results showed that NB84 suppressed nonsense mutation more efficiently than the other compounds. ${ }^{63}$ In vivo treatment reduced urine GAGs excretion and tissue GAGs storage, showing that this option may have therapeutic efficacy. Although preclinical results are not always reproduced in clinical trials (as occurred with SCRT for Duchenne muscular dystrophy), SCRT may be a potential therapeutic strategy for MPS treatment with the advantage that the drugs used in this approach, as with the drugs used for substrate reduction and chaperone therapies, can be administered orally and are able to cross the BBB. This represents an opportunity for treatment of patients with neurological symptoms.

\section{Gene therapy}

The discovery that lysosomal enzymes can be secreted and taken up by adjacent or distant cells via the M6P receptor, thereby bringing about cross-correction, made gene therapy especially attractive for the MPS disorders. Figure 1 shows the rationale for gene therapy in the MPS. Both in vivo and ex vivo protocols using different vectors have been successfully tested in animal models. ${ }^{64,65}$

Some nonviral approaches have been tested for treatment of MPS. Simple hydrodynamic plasmid injections through the tail vein resulted in partial correction in the MPS I mouse model, ${ }^{66}$ and even better results were achieved in MPS VII mice. ${ }^{67}$ However, the transient expression of the transgene made researchers look for other nonviral methods of gene

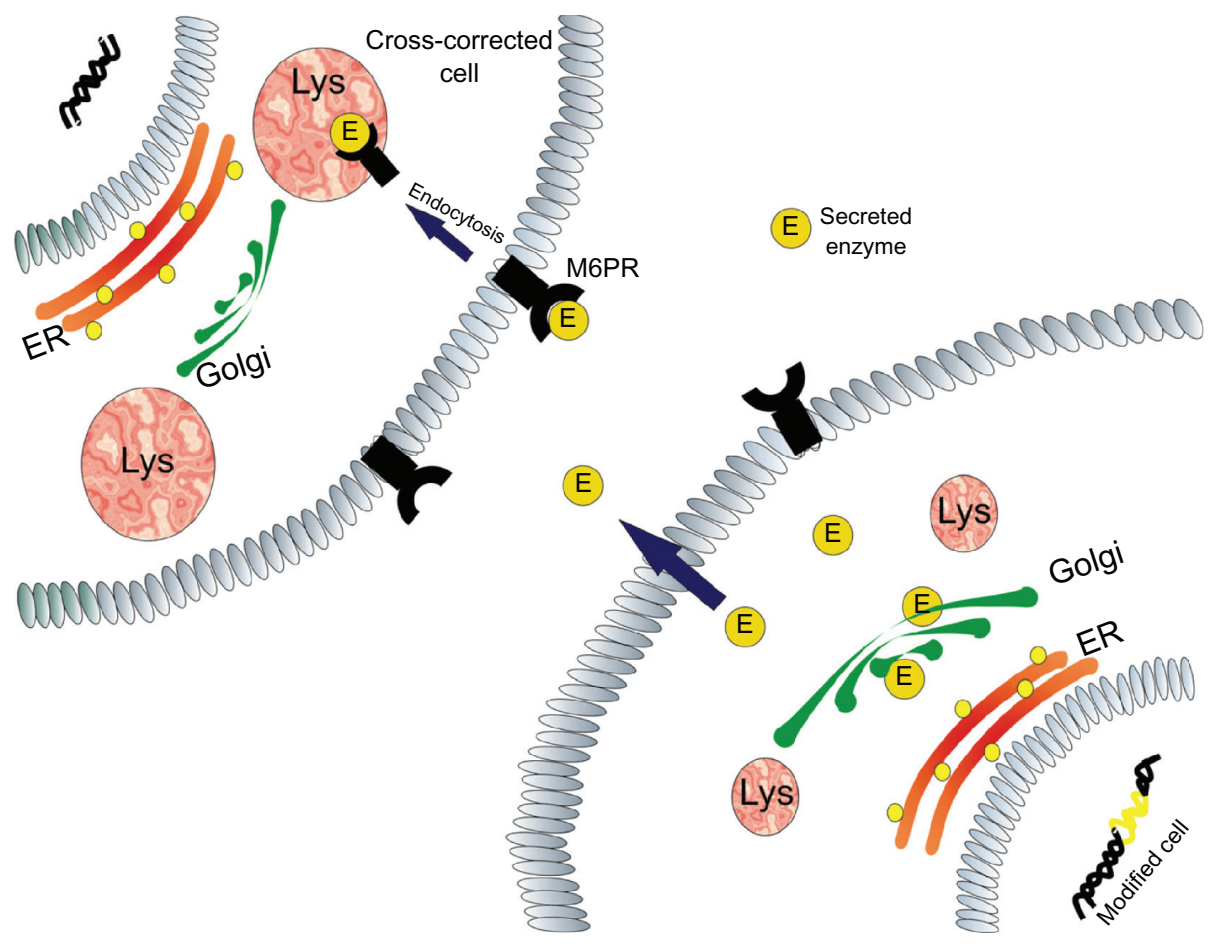

Figure I Rationale for gene therapy in MPS disorders.

Notes: The genetically modified cells (at the bottom) produce a normal copy of the enzyme, which is secreted to the extracellular media and is able to be internalized by adjacent cells using the M6PR. The internalized enzyme reduces the glycosaminoglycan storage in the lysosome.

Abbreviations: E, enzyme; M6PR, mannose-6-phosphate receptor; ER, endoplasmic reticulum; MPS, mucopolysaccharidoses. 
transfer. In that context, removal of some bacterial sequences from the construct coupled with immunomodulation allowed for long-term expression of IDUA in MPS I mice. ${ }^{68}$ Another interesting approach used the Sleeping Beauty transposon system to allow transgene integration into host cells. IDUA activity in both visceral tissues as well as in the brain was detected after Sleeping Beauty-mediated gene therapy in MPS I mice. ${ }^{69}$ To confer protection against the immune system, cell lines were transfected with plasmids and encapsulated into polymer membranes. This approach has shown benefits in correcting both MPS I cells, ${ }^{70}$ as well as MPS I, ${ }^{71,72}$ MPS II ${ }^{73}$ and MPS VII ${ }^{74}$ mice when applied intraperitoneally. MPS VII mice treated with an intraventricular injection of the capsules have also shown improvements in behavioral and biochemical abnormalities,${ }^{75}$ confirming that this strategy is a potential treatment for the MPS.

However, it should be noted that most gene therapy protocols for MPS include modified viruses as vectors due to their higher efficiency of transduction and long-term gene expression. These vectors have been applied both in situ and systemically, depending on the characteristics of the MPS studied. Most viral vectors used for MPS are derived from retroviruses, lentiviruses, and adeno-associated viruses (AAV).

The retroviral vectors have an RNA genome that is copied to DNA and integrates into chromosomes, producing long-term expression. Their main disadvantages include the fact that they only transduce dividing cells and they can produce insertional mutagenesis. ${ }^{76}$ Protocols using a gammaretrovirus for MPS I and MPS VII resulted in high and stable serum IDUA and GUSB levels, and they complete corrections in both mouse ${ }^{64,77-79}$ and dog models. ${ }^{77}$

Lentiviral vectors are a type of retroviral vector that is able to transduce nondividing cells. Due to this property, they are suitable for the treatment of diseases affecting the CNS. In situ delivery of lentiviral vectors has proven to be effective in treating brain abnormalities found in MPS IIIA, ${ }^{80}$ MPS IIIB,${ }^{81}$ and MPS VII ${ }^{82}$ mice. Furthermore, systemically injected lentiviruses also had a positive outcome on somatic and brain diseases. ${ }^{82}$

In recent years, several studies have tested different serotypes of AAV vectors for gene therapy in MPS. These vectors have a single stranded DNA genome, which is converted into a double-stranded DNA that is able to form concatemers in the nucleus, leading to long-term gene expression; integration into the chromosome occurs occasionally. Several studies have addressed the efficacy of using AAV vectors for different types of MPS. AAV-8 gene therapy has been shown to effectively transduce liver cells and ameliorate somatic and brain abnormalities in MPS IIIA mice when applied intravenously. ${ }^{83}$ An intravenous injection of a AAV2/8 vector was able to ameliorate the clinical signs found in MPS VI cats for up to 1 year. ${ }^{84}$ Combining an intravenous and an intracisternal injection of AAV-2 resulted in increased lifespan and correction of brain abnormalities in MPS IIIB mice. ${ }^{85}$ Furthermore, injections of AAV-5.5 or AAV-2.5 in dog models of MPS I and IIIB have shown long-term correction of abnormalities without serious adverse effects. ${ }^{86}$

Other studies have used gene therapy in combination with other therapies as complementary approaches. A study on MPS IIIB mice ${ }^{87}$ evaluated bone marrow transplant alone, intracranial AAV2/5 gene therapy alone, or the combination of both. Combination therapy, compared to either therapy alone, resulted in synergistic effects on hearing as well as CNS lysosomal inclusions; however, antagonistic effects were noted on motor function and lifespan. A second study on MPS I mice compared hematopoietic stem cell transplantation alone (without genetic modifications) or with hematopoietic stem cells transduced with a lentiviral vector. ${ }^{66}$ The ex vivo gene therapy approach was able to correct brain and skeletal abnormalities in MPS I mice, which were not corrected in the group treated with nontransduced cells. The authors concluded that the gene therapy was able to augment the efficacy of the stem cell transplant.

Gene therapy also faces problems with the distribution of the vector in the brain, which might be a challenge in larger brains like those found in humans. In addition, we need to learn more about the long-term effects of gene therapy, as the inserted genes would work for decades in humans. With the improvements in vector designs and delivery methods, it is likely that this approach will be tested in the near future, and possibly across different types of MPS. Clinical trials with gene therapy for MPS III types are currently being planned.

\section{Neural stem cells}

Neural stem cells (NSC) have been isolated from human and murine brains. These cells possess the capacity to grow indefinitely and the potential to differentiate into three major cell types of CNS cells: neurons, astrocytes, and oligodendrocytes. ${ }^{88}$ The unique properties of NSC include their ability to migrate to distal sites, differentiate into various cell types, and to integrate within the host brain without disrupting normal function. ${ }^{89}$ Therefore, these cells are attractive as delivery vehicles not only for replacing the deficient enzymes in the MPS but also to restore neurological deficits present in these diseases. 
In the mouse model of MPS VII, genetically engineered mouse NSC overexpressing GUSB were transplanted into the cerebral ventricles of newborn mice. At maturity, donorderived cells were present as normal constituents of diverse brain regions, and GUSB activity was detected along the entire neuroaxis, resulting in widespread correction of lysosomal storage in the mouse brain..$^{90}$ Meng et al ${ }^{91}$ retrovirally transduced a neural stem cell line derived from human embryonic telencephalon to overexpress GUSB. Cells were transplanted into the cerebral ventricles of neonatal MPS VII mice; 25 days after implant, GAGs storage in the brain was found to be significantly reduced in the transplanted mice. This study provided evidence that human neural stem cells would serve as a useful gene transfer vehicle for the treatment of diffuse CNS alterations in LSDs. ${ }^{92}$ Another research group used fetal neural stem cells derived from embryonic mouse striata, and these were expanded in vitro by neurosphere formation. Neurospheres were then transplanted into the lateral ventricles of newborn MPS VII mice, and donor cells migrated far beyond the site of injection within 24 hours. GUSB activity showed a slight increase; however, histological analysis revealed a widespread decrease in lysosomal storage in the recipient's hippocampus, cortex, and ependyma. In addition, functional assessment with novel-object recognition tests confirmed improvements in behavioral patterns. ${ }^{93}$

Despite encouraging results in animal models, there are a number of issues to be clarified before NSC as a stem cell-based therapy can be applied in the clinical setting. First, it should be noted that these studies incorporated a murine model; the mouse brain is small, thus allowing for the widespread diffusion of lysosomal enzymes. ${ }^{94}$ Second, the long-term effects are unknown, as mouse experiments are limited to months rather than to the human requirement of years. ${ }^{94}$ Finally, the source of the cells is also a matter of concern. In this sense, induced pluripotent stem cells have major potential advantages because patient-specific neuroblasts are suitable for transplantation, they avoid immune reactions, and they can be produced without the use of human embryonic stem cells. ${ }^{95}$ Although induced pluripotent stem cells have not yet been successfully used in clinical trials, different groups have been successful in generating such cells from MPS VII and MPS IIIB patients. ${ }^{96,97}$ So far, the only investigational application that has been developed to assess the use of human NSC allografts in metabolic disorders was specifically targeted for treating Batten's disease (NCL2), and this therapy seems to have been well tolerated by patients. ${ }^{98}$ Therefore, this seems to be a promising strategy for the MPS with neurological involvement.

Table I Available and emerging therapies for the mucopolysaccharidoses*

\begin{tabular}{|c|c|c|c|c|c|c|c|}
\hline MPS & Intravenous ERT & Intrathecal ERT & SRT & Chaperones & SCRT & Gene therapy & NSC \\
\hline $\mathrm{I}$ & Available & $\begin{array}{l}\text { Pre-clinical studies } \\
\text { published; case } \\
\text { report published }\end{array}$ & - & - & $\begin{array}{l}\text { Preclinical } \\
\text { studies } \\
\text { published }\end{array}$ & $\begin{array}{l}\text { Preclinical studies } \\
\text { published }\end{array}$ & - \\
\hline II & Available & $\begin{array}{l}\text { Clinical trial } \\
\text { in progress }\end{array}$ & $\begin{array}{l}\text { Preclinical and clinical } \\
\text { studies published }\end{array}$ & - & - & $\begin{array}{l}\text { Preclinical studies } \\
\text { published }\end{array}$ & - \\
\hline IIIA & - & $\begin{array}{l}\text { Clinical trial } \\
\text { in progress }\end{array}$ & $\begin{array}{l}\text { Preclinical and clinical } \\
\text { studies published }\end{array}$ & - & - & $\begin{array}{l}\text { Preclinical studies } \\
\text { published clinical } \\
\text { trial in progress }\end{array}$ & - \\
\hline IIIB & - & - & $\begin{array}{l}\text { Preclinical and clinical } \\
\text { studies published }\end{array}$ & In vitro studies & - & $\begin{array}{l}\text { Preclinical studies } \\
\text { published }\end{array}$ & - \\
\hline IIIC & - & - & - & In vitro studies & - & - & - \\
\hline IIID & - & - & - & - & - & - & - \\
\hline IVA & $\begin{array}{l}\text { Clinical trial } \\
\text { in progress }\end{array}$ & - & - & - & - & - & - \\
\hline IVB & - & - & - & In vitro studies & - & - & - \\
\hline VI & Available & $\begin{array}{l}\text { Case report } \\
\text { published }\end{array}$ & - & - & - & $\begin{array}{l}\text { Preclinical studies } \\
\text { published }\end{array}$ & - \\
\hline VII & $\begin{array}{l}\text { Preclinical studies } \\
\text { ongoing; clinical } \\
\text { trial planned }\end{array}$ & - & - & - & - & $\begin{array}{l}\text { Preclinical studies } \\
\text { published }\end{array}$ & $\begin{array}{l}\text { Preclinical studies } \\
\text { published }\end{array}$ \\
\hline IX & - & - & - & - & - & - & - \\
\hline
\end{tabular}

Note: *This table does not include bone marrow transplantation/hematopoietic stem cell transplantation. HSCT is indicated before 2.5 years of age in severe MPS I, to preserve cognitive function.

Abbreviations: ERT, enzyme replacement therapy; SRT, substrate reduction therapy; SCRT, stop-codon read-through; NSC, neural stem cells. 


\section{Conclusion}

Over the last decade a whole new set of therapeutic measures have become available or are in development to address the MPS. Intravenous ERT, already approved for MPS I, II, and VI, will possibly be available for both MPS IVA and MPS VII within the next few years. Intrathecal ERT, tested in animals and already reported in a few patients, may become a tool to treat or prevent the CNS manifestations of these diseases. Currently, clinical developments are underway for the severe form of MPS I (associated or not to BMT/ HSCT), and for MPS II, MPS IIIA, and possibly for MPS IIIB. Substrate inhibition therapy with small molecules which cross the BBB is also being tested for the MPS types with CNS manifestations (mainly MPS III, but also for MPS II). In vitro studies point out that chaperones may also be a therapeutic tool, with preliminary data indicating that they may be useful in MPS IIIB, MPS IIIC and MPS IVB. SCRT strategy has been tried in preclinical studies for MPS I, and may be a tool to attenuate the clinical manifestations of the severe form of this disease when caused by nonsense mutations. Despite the number of preclinical studies that were conducted to investigate gene therapy, and presented with quite encouraging results, this treatment modality took a long time to move forward into clinical studies; however, it should be noted that a trial for MPS IIIA should begin soon. The use of neural stem cells in the MPS types which have CNS involvement is promising, and more experimental results should become available in the next few years. Table 1 summarizes the present situation regarding the available and emerging options for the MPS diseases.

The MPS are hard-to-treat diseases, which demand, in addition to the available specific therapies, a broad range of supportive measures that are very important to improve the patient's quality of life. ${ }^{99}$ As expected, most of the technology-intensive therapies for rare and ultra-rare diseases have very high costs, which brings an extra challenge for access to these treatments.

Another important point to mention is that early diagnosis followed by early treatment seems to considerably improve patient outcomes. ${ }^{100}$ Since there is currently a significant delay in MPS diagnosis after the onset of symptoms, ${ }^{101,102}$ it is important to establish early diagnoses to prevent disease progression; in this light, newborn screening for these diseases (already technically possible) may be considered as a wider set of treatment possibilities become available for these diseases.

\section{Acknowledgment}

The authors express their gratitude to Dr Anthony H Fensom for the careful review of the text and for his valuable suggestions.

\section{Disclosure}

The authors report no conflicts of interest in this work.

\section{References}

1. Neufeld E, Muenzer J. The mucopolysaccharidosis. In: Scriver CR, Beaudet AL, Sly WS, Valle D, editors. The Metabolic and Molecular Basis of Inherited Disease. New York: McGraw-Hill; 2001:3421-3451.

2. Giugliani R, Federhen A, Munoz Rojas MV, et al. [Enzyme replacement therapy for mucopolysaccharidoses I, II and VI: recommendations from a group of Brazilian experts]. RevAssoc Med Bras. 2010;56(3):257-277. Portuguese.

3. Beck M. Therapy for lysosomal storage disorders. IUBMB Life. 2010;62(1):33-40

4. Valayannopoulos V, Wijburg FA. Therapy for the mucopolysaccharidoses. Rheumatology (Oxford). 2011;50 Suppl 5:v49-v59.

5. Clarke LA, Wraith JE, Beck M, et al. Long-term efficacy and safety of laronidase in the treatment of mucopolysacchoaridosis I. Pediatrics. 2009;123(1):229-240.

6. Wraith JE, Scarpa M, Beck M, et al. Mucopolysaccharidosis type II (Hunter syndrome): a clinical review and recommendations for treatment in the era of enzyme replacement therapy. Eur J Pediatr. 2008; 167(3):267-277.

7. Harmatz P, Giugliani R, Schwartz IV, et al; MPS VI Study Group. Long-term follow-up of endurance and safety outcomes during enzyme replacement therapy for mucopolysaccharidosis VI: Final results of three clinical studies of recombinant human $\mathrm{N}$-acetylgalactosamine 4-sulfatase. Mol Genet Metab. 2008;94(4):469-475.

8. Dickson P, Peinovich M, McEntee M, et al. Immune tolerance improves the efficacy of enzyme replacement therapy in canine mucopolysaccharidosis I. J Clin Invest. 2008;118(8):2868-2876.

9. Horovitz D, Magalhães TSPC, Bonfim C, et al. Enzyme replacement therapy (ERT) and hematopoietic stem cell transplantation (HSCT) in a very young child with Hunter Syndrome: two year follow-up. Abstract: Proceedings of the 12th International Symposium on MPS and related diseases; June 28-July 1, 2012; Noordwijkerhout, The Netherlands: p. 103.

10. Tomatsu S, Montaño AM, Oikawa $\mathrm{H}$, et al. Mucopolysaccharidosis type IVA (Morquio A disease): clinical review and current treatment Curr Pharm Biotechnol. 2011;12(6):931-945.

11. Kakkis ED, Neufeld EF. The mucopolysaccharidoses. In: Berg BO, editor. Principles of Child Neurology. New York: McGraw-Hill, 1996:1141-1165.

12. Dvorak-Ewell M, Wendt D, Hague C, et al. Enzyme replacement in a human model of mucopolysaccharidosis IVA in vitro and its biodistribution in the cartilage of wild type mice. PLoS One. 16, 2010;5(8):e12194.

13. Tomatsu S, Montaño AM, Dung VC, et al. Enhancement of drug delivery: enzyme-replacement therapy for murine Morquio A syndrome. Mol Ther. 2010;18(6):1094-1102.

14. BioMarin. BioMarin provides update on GALNS Phase 1/2 Extension Study (MOR-100) [press release]. Novato, CA: Biomarin Pharmaceutical Inc; July 28, 2011. Available from: http://phx.corporate-ir.net/phoenix. $\mathrm{zhtml}$ c $=106657 \& \mathrm{p}=$ irol-newsArticle $\&$ ID $=1590497 \&$ highlight $=$ Accessed February 23, 2012.

15. Sly WS, Quinton BA, McAlister WH, Rimoin DL. Beta glucuronidase deficiency: report of clinical, radiologic, and biochemical features of a new mucopolysaccharidosis. J Pediatr. 1973;82(2):249-257.

16. Sly WS. Enzyme replacement therapy: from concept to clinical practice. Acta Paediatr Suppl. 2002;91(439):71-78. 
17. Vogler C, Levy B, Grubb JH, et al. Overcoming the blood-brain barrier with high-dose enzyme replacement therapy in murine mucopolysaccharidosis VII. Proc Natl Acad Sci U S A. 2005;102(41):14777-14782.

18. National MPS Society. A Guide to Understanding MPS VII. Durham, NC: National MPS Society; 2008. Available from: http://www. mpssociety.org/wp-content/uploads/2011/07/MPS_VII_2008.pdf. Accessed February 23, 2012.

19. Chen AH, Dickson PI. Enzyme replacement therapy for cognitive decline in mucopolysaccharidosis Type I: past, present, and future. Lysosomal Storage Diseases. 2011;9(1):9-16.

20. Auclair D, Finnie J, White J, et al. Repeated intrathecal injections of recombinant human-4-sulphatase remove dural storage in mature mucopolysaccharidosis VI cats primed with a short-course tolerisation regimen. Mol Gen Metab. 2010;99(2):132-141.

21. Dickson PI, Hanson S, McEntee MF, et al. Early versus late treatment of spinal cord compression with long-term intrathecal enzyme replacement therapy in canine mucopolysaccharidosis type I. Mol Genet Metab. 2010;101(2-3):115-122.

22. Kakkis E, Lester T, Yang R, et al. Successful induction of immune tolerance to enzyme replacement therapy in canine mucopolysaccharidosis I. Proc Natl Acad Sci U S A. 2004;101(3):829-834.

23. Munoz-Rojas MV, Vieira T, Costa R, et al. Intrathecal enzyme replacement therapy in a patient with mucopolysaccharidosis type I and symptomatic spinal cord compression. Am J Med Genet A. 2008;146A(19):2538-2544.

24. Munoz-Rojas MV, Horovitz DD, Jardim LB, et al. Intrathecal administration of recombinant human $\mathrm{N}$-acetylgalactosamine 4-sulfatase to a MPS VI patient with pachymeningitis cervicalis. Mol Genet Metab. 2010;99(4):346-350.

25. Jakóbkiewicz-Banecka J, Wegrzyn A, Wegrzyn G. Substrate deprivation therapy: a new hope for patients suffering from neuronopathic forms of inherited lysosomal storage diseases. J Appl Genet. 2007;48(4): 383-388.

26. Roberts AL. Substrate Deprivation as a Novel Therapy for the Mucopolysaccharidoses [doctoral thesis]. South Australia: University of Adelaide; 2007.

27. Piotrowska E, Jakóbkiewicz-Banecka J, Baranska S, et al. Genisteinmediated inhibition of glycosaminoglycan synthesis as a basis for gene expression-targeted isoflavone therapy for mucopolysaccharidoses. Eur J Hum Genet. 2006;14(7):846-852.

28. Friso A, Tomanin R, Salvalaio M, Scarpa M. Genistein reduces glycosaminoglycan levels in a mouse model of mucopolysaccharidosis type II. Br J Pharmacol. 2010;159(5):1082-1091.

29. Malinowska M, Wilkinson FL, Bennett W, et al. Genistein reduces lysosomal storage in peripheral tissues of mucopolysaccharide IIIB mice. Mol Genet and Metab. 2009;98(3):235-242.

30. Malinowska M, Wilkinson FL, Langford-Smith KJ, et al. Genistein improves neuropathology and corrects behaviour in a mouse model of neurodegenerative metabolic disease. PLoS One. 2010;5(12): e14192.

31. Piotrowska E, Jakóbkiewicz-Banecka J, Tylki-Szymanska A, et al. Genistin-rich soy isoflavone extract in substrate reduction therapy for Sanfilippo syndrome: an open-label, pilot study in 10 pediatric patients. Current Therapeutic Research. 2008;69(2):166-179.

32. Tsai TH. Concurrent measurement of unbound genistein in the blood, brain and bile of anesthetized rats using microdialysis and its pharmacokinetic application. J Chromatogr A. 2005;1073(1-2):317-322.

33. Akiyama T, Ishida J, Nakagawa $\mathrm{S}$, et al. Genistein, a specific inhibitor of tyrosine-specific protein kinases. J Biol Chem. 1987;262(12): $5592-5595$.

34. KloskaA,Jakóbkiewicz-Banecka J, Narajczyk M, Banecka-MajkutewiczZ, Wegrzyn G. Effects of flavonoids on glycosaminoglycan synthesis: implications for substrate reduction therapy in Sanfilippo disease and other mucopolysaccharidoses. Metab Brain Dis. 2011;26(1):1-8.

35. Wijburg FA, Valstar MJ, Ruijter de J. Genistein: results of clinical trials. Proceedings of the 12th International Symposium on MPS and related diseases; June 28-July 1, 2012; Noordwijkerhout, The Netherlands: p. 43.
36. Bigger B. Genistein: animal studies and a new trial. 12th International Symposium on MPS and related diseases. Proceedings of the 12th International Symposium on MPS and related diseases; June 28-July 1, 2012; Noordwijkerhout, The Netherlands: p. 44.

37. Kim K, Dodsworth C, Paras A, Burton BK. High dose synthetic genistein treatment in MPS patients with neurological involvement. Proceedings of the 12th International Symposium on MPS and related diseases; June 28-July 1, 2012; Noordwijkerhout, The Netherlands: p. 76.

38. Kaji T, Kawashima T, Sakamoto M. Rhodamine B inhibition of glycosaminoglycan production by cultured human lip fibroblasts. Toxicol Appl Pharmacol. 1991;111(1):82-89.

39. Roberts AL, Thomas BJ, Wilkinson AS, Fletcher JM, Byers S. Inhibition of glycosaminoglycan synthesis using rhodamine B in a mouse model of mucopolysaccharidosis type IIIA. Pediatr Res. 2006;60(3):309-314.

40. Roberts AL, Rees MH, Klebe S, Fletcher JM, Byers S. Improvement in behaviour after substrate deprivation therapy with rhodamine $B$ in a mouse model of MPS IIIA. Mol Genet Metab. 2007;92(1-2):115-121.

41. Roberts AL, Fletcher JM, Moore L, Byers S. Trans-generational exposure to low levels of rhodamine B does not adversely affect litter size or liver function in murine mucopolysaccharidosis type IIIA. Mol Genet Metab. 2010;101(2-3):208-213.

42. Fan JQ, Ishii S. Active-site-specific chaperone therapy for Fabry disease. Yin and Yang of enzyme inhibitors. FEBS J. 2007;274(19): 4962-4971.

43. Parenti G. Treating lysosomal storage diseases with pharmacological chaperones: from concept to clinics. EMBO Mol Med. 2009;1(5): 268-279.

44. Schiffmann R. Therapeutic approaches for neuronopathic lysosomal storage disorders. J Inherit Metab Dis. 2010;33(4):373-379.

45. Lim-Melia ER, Kronn DF. Current enzyme replacement therapy for the treatment of lysosomal storage diseases. Pediatr Ann. 2009;38(8): 448-455.

46. Valenzano KJ, Khanna R, Powe AC, et al. Identification and characterization of pharmacological chaperones to correct enzyme deficiencies in lysosomal storage disorders. Assay Drug Dev Technol. 2011;9(3):213-235.

47. Fan JQ, Ishii S, Asano N, Suzuki Y. Accelerated transport and maturation of lysosomal alpha-galactosidase A in Fabry lymphoblasts by an enzyme inhibitor. Nat Med. 1999;5(1):112-115.

48. Germain DP, Fan JQ. Pharmacological chaperone therapy by active-sitespecific chaperones in Fabry disease: in vitro and preclinical studies. Int J Clin Pharmacol Ther. 2009;47 Suppl 1:S111-S117.

49. Beck M. Emerging drugs for lysosomal storage diseases. Expert Opin Emerg Drugs. 2010;15(3):495-507.

50. Loo TW, Clarke DM. Chemical and pharmacological chaperones as new therapeutic agents. Expert Rev Mol Med. 2007;9(16):1-18.

51. Porto C, Cardone M, Fontana F, et al. The pharmacological chaperone N-butyldeoxynojirimycin enhances enzyme replacement therapy in Pompe disease fibroblasts. Mol Ther. 2009;17(6):964-971.

52. de Ruijter J, Valstar MJ, Wijburg FA. Mucopolysaccharidosis type III (Sanfilippo Syndrome): emerging treatment strategies. Curr Pharm Biotechnol. 2011;12(6):923-930.

53. Feldhammer M, Durand S, Pshezhetsky AV. Protein misfolding as an underlying molecular defect in mucopolysaccharidosis III type C. PLoS One. 2009;4(10): 7434

54. Suzuki Y, Ogawa S, Sakakibara Y. Chaperone therapy for neuronopathic lysosomal diseases: competitive inhibitors as chemical chaperones for enhancement of mutant enzyme activities. Perspect Medicin Chem. 2009;3:7-19.

55. Wilschanski M, Yahav Y, Yaacov Y, et al. Gentamicin-induced correction of CFTR function in patients with cystic fibrosis and CFTR stop mutations. N Engl J Med. 2003;349(15):1433-1441.

56. Linde L, Kerem B. Introducing sense into nonsense in treatments of human genetic diseases. Trends Genet. 2008;24(11):552-563.

57. Keeling KM, Bedwell DM. Clinically relevant aminoglycosides can suppress disease-associated premature stop mutations in the IDUA and P53 cDNAs in a mammalian translation system. J Mol Med (Berl). 2002;80(6):367-376. 
58. Hein LK, Bawden M, Muller VJ, Sillence D, Hopwood JJ, Brooks DA. Alpha-L-iduronidase premature stop codons and potential readthrough in mucopolysaccharidosis type I patients. $J$ Mol Biol. 2004;338(3):453-462.

59. Menon KP, Neufeld EF. Evidence for degradation of mRNA encoding alpha-L-iduronidase in Hurler fibroblasts with premature termination alleles. Cell Mol Biol (Noisy-le-grand). 1994;40(7):999-1005.

60. Lualdi S, Di Rocco M, Corsolini F, et al. Identification of nine new IDS alleles in mucopolysaccharidosis II. Quantitative evaluation by real-time RT-PCR of mRNAs sensitive to nonsense-mediated and nonstop decay mechanisms. Biochim Biophys Acta. 2006;1762(4):478-484.

61. Linde L, Boelz S, Nissim-Rafinia M, et al. Nonsense-mediated mRNA decay affects nonsense transcript levels and governs response of cystic fibrosis patients to gentamicin. $J$ Clin Invest. 2007;117(3): 683-692.

62. Keeling KM, Brooks DA, Hopwood JJ, Li P, Thompson JN, Bedwell DM. Gentamicin-mediated suppression of Hurler syndrome stop mutations restores a low level of alpha-L-iduronidase activity and reduces lysosomal glycosaminoglycan accumulation. Hum Mol Genet. 2001;10(3): 291-299.

63. Wang D, Belakhov V, Kandasamy J, et al. The designer aminoglycoside NB84 significantly reduces glycosaminoglycan accumulation associated with MPS I-H in the Idua-W392X mouse. Mol Genet Metab. 2012;105(1): $116-125$

64. Chung S, Ma X, Liu Y, Lee D, Tittiger M, Ponder KP. Effect of neonatal administration of a retroviral vector expressing alpha-L-iduronidase upon lysosomal storage in brain and other organs in mucopolysaccharidosis I mice. Mol Genet Metab. 2007;90(2):181-192.

65. Visigalli I, Delai S, Politi LS, et al. Gene therapy augments the efficacy of hematopoietic cell transplantation and fully corrects mucopolysaccharidosis type I phenotype in the mouse model. Blood. 2010;116(24):5130-5139.

66. Camassola M, Braga LM, Delgado-Cañedo A, et al. Nonviral in vivo gene transfer in the mucopolysaccharidosis I murine model. $J$ Inherit Metab Dis. 2005;28(6):1035-1043.

67. Richard M, Arfi A, Seguin J, Gandolphe C, Scherman D. Widespread biochemical correction of murine mucopolysaccharidosis type VII pathology by liver hydrodynamic plasmid delivery. Gene Ther. 2009, 16(6):746-756.

68. Osborn MJ, McElmurry RT, Lees CJ, et al. Minicircle DNA-based gene therapy coupled with immune modulation permits long-term expression of $\alpha$-L-iduronidase in mice with mucopolysaccharidosis type I. Mol Ther. 2011;19(3):450-460.

69. Aronovich EL, Bell JB, Khan SA, et al. Systemic correction of storage disease in MPS I NOD/SCID mice using the sleeping beauty transposon system. Mol Ther. 2009;17(7):1136-1144.

70. Baldo G, Quoos Mayer F, Burin M, Carrillo-Farga J, Matte U, Giugliani R. Recombinant encapsulated cells overexpressing alpha-L-iduronidase correct enzyme deficiency in human mucopolysaccharidosis type I cells. Cells Tissues Organs. 2012;195(4):323-329.

71. Piller Puicher E, Tomanin R, Salvalaio M, et al. Encapsulated engineered myoblasts can cure Hurler syndrome: preclinical experiments in the mouse model. Gene Therapy. 2012;19(4):355-364.

72. Baldo G, Mayer FQ, Martinelli B, et al. Intraperitoneal implant of recombinant encapsulated cells overexpressing alpha-1-iduronidase partially corrects visceral pathology in mucopolysaccharidosis type I mice. Cytotherpy. 2012;14(7):860-867.

73. Friso A, Tomanin R, Alba S, et al. Reduction of GAG storage in MPS II mouse model following implantation of encapsulated recombinant myoblasts. J Gene Med. 2005; 7(11):1482-1491.

74. Ross CJ, Bastedo L, Maier SA, Sands MS, Chang PL. Treatment of a lysosomal storage disease, mucopolysaccharidosis VII, with microencapsulated recombinant cells. Hum Gene Ther. 2000;11(15): 2117-2127.

75. Ross CJ, Ralph M, Chang PL. Somatic gene therapy for a neurodegenerative disease using microencapsulated recombinant cells. Exp Neurol. 2000;166(2):276-286
76. Hacein-Bey-Abina S, von Kalle C, Schmidt M, et al. A serious adverse event after successful gene therapy for X-linked severe combined immunodeficiency. N Engl J Med. 2003;348(3):255-256.

77. Mango RL, Xu L, Sands MS, et al. Neonatal retroviral vector-mediated hepatic gene therapy reduces bone, joint, and cartilage disease in mucopolysaccharidosis VII mice and dogs. Mol Genet Metab. 2004;82(1):4-19.

78. Baldo G, Wu S, Howe RA, et al. Pathogenesis of aortic dilatation in mucopolysaccharidosis VII mice may involve complement activation. Mol Genet Metab. 2011;104(4):608-619.

79. Herati RS, Ma X, Tittiger M, Ohlemiller KK, Kovacs A, Ponder KP. Improved retroviral vector design results in sustained expression after adult gene therapy in mucopolysaccharidosis I mice. J Gene Med. 2008;10(9):972-982.

80. McIntyre C, Derrick Roberts AL, Ranieri E, Clements PR, Byers S, Anson DS. Lentiviral-mediated gene therapy for murine mucopolysaccharidosis type IIIA. Mol Genet Metab. 2008;93(4): 411-418.

81. Di Natale P, Di Domenico C, Di Napoli D. Serum MIP-1 alpha level: a biomarker for the follow-up of lentiviral therapy in mucopolysaccharidosis IIIB mice. J Inherit Metab Dis. 2010;33(2):159-165.

82. Bielicki J, McIntyre C, Anson DS. Comparison of ventricular and intravenous lentiviral-mediated gene therapy for murine MPS VII. Mol Genet Metab. 2010;101(4):370-382.

83. Ruzo A, Garcia M, Ribera A, et al. Liver production of sulfamidase reverses peripheral and ameliorates CNS pathology in mucopolysaccharidosis IIIA mice. Mol Ther. 2012;20(2):254-266.

84. Cotugno G, Annunziata P, Tessitore A, et al. Long-term amelioration of feline Mucopolysaccharidosis VI after AAV-mediated liver gene transfer. Mol Ther. 2011;19(3):461-469.

85. Fu H, Kang L, Jennings JS, et al. Significantly increased lifespan and improved behavioral performances by rAAV gene delivery in adult mucopolysaccharidosis IIIB mice. Gene Ther. 2007;14(14): 1065-1077.

86. Ellinwood NM, Ausseil J, Desmaris N, et al. Safe, efficient, and reproducible gene therapy of the brain in the dog models of Sanfilippo and Hurler syndromes. Mol Ther. 2011;19(2):251-259.

87. Heldermon CD, Ohlemiller KK, Herzog ED, et al. Therapeutic efficacy of bone marrow transplant, intracranial AAV-mediated gene therapy, or both in the mouse model of MPS IIIB. Mol Ther. 2010;18(5):873-880.

88. Kim S. Genetically engineered human neural stem cells for brain repair in neurological diseases. Brain Dev. 2007;29(4):193-201.

89. Shihabuddin LS, Cheng SH. Neural stem cell transplantation as a therapeutic approach for treating lysosomal storage diseases. Neurotherapeutics. 2011;8(4):659-667.

90. Snyder EY, Taylor RM, Wolfe JH. Neural progenitor cell engraftment corrects lysosomal storage throughout the MPS VII mouse brain. Nature. 1995;374(6520):367-370.

91. Meng XL, Shen JS, Ohashi T, Maeda H, Kim S, Eto Y. Brain transplantation of genetically engineered human neural stem cells globally corrects brain lesions in the mucopolysaccharidosis type VII mouse. $J$ Neurosci Res. 2003;74(2):266-277.

92. Eto Y, Shen JS, Meng XL, Ohashi T. Treatment of lysosomal storage disorders: cell therapy and gene therapy. J Inherit Metab Dis. 2004;27(3):411-415.

93. Fukuhara Y, Li XK, Kitazawa Y, et al. Histopathological and behavioral improvement of murine mucopolysaccharidosis type VII by intracerebral transplantation of neural stem cells. Mol Ther. 2006;13(3):548-555.

94. Sidman RL, Li J, Stewart GR, Clarke J, Yang W, Snyder EY, Shihabuddin LS. Injection of mouse and human neural stem cells into neonatal Niemann-Pick A model mice. Brain Res. 2007;1140:195-204.

95. Feng Z, Gao F. Stem cell challenges in the treatment of neurodegenerative disease. CNS Neurosci Ther. 2012;18(2):142-148.

96. Meng XL, Shen JS, Kawagoe S, Ohashi T, Brady RO, Eto Y. Induced pluripotent stem cells derived from mouse models of lysosomal storage disorders. Proc Natl Acad Sci U SA. 2010;107(17): 7886-7891. 
97. Lemonnier T, Blanchard S, Toli D, et al. Modeling neuronal defects associated with a lysosomal disorder using patient-derived induced pluripotent stem cells. Hum Mol Genet. 2011;20(18):3653-3666.

98. Kohan R, Cismondi IA, Oller-Ramirez AM, et al. Therapeutic approaches to the challenge of neuronal ceroid lipofuscinoses. $\mathrm{Curr}$ Pharm Biotechnol. 2011;12(6):867-883.

99. Giugliani R, Federhen A, Muñoz Rojas MV, et al. Mucopolysaccharidosis I, II, and VI: Brief review and guidelines for treatment. Genet Mol Biol. 2010;33(4):589-604.

100. McGill JJ, Inwood AC, Coman DJ, et al. Enzyme replacement therapy for mucopolysaccharidosis VI from 8 weeks of age - a sibling control study. Clin Genet. 2010;77(5):492-498.
101. Vieira T, Schwartz I, Muñoz V, et al. Mucopolysaccharidoses in Brazil: what happens from birth to biochemical diagnosis? Am JMed Genet A. 2008;146A(13):1741-1747.

102. Alonso-Fernández JR, Fidalgo J, Colón C. Neonatal screening for mucopolysaccharidoses by determination of glycosaminoglycans in the eluate of urine-impregnated paper: preliminary results of an improved DMB-based procedure. J Clin Lab Anal. 2010;24(3):149-153.

\section{Publish your work in this journal}

Research and Reports in Endocrine Disorders is an international, peerreviewed, open access journal publishing original research, reports, reviews and commentaries on all areas of endocrinology, endocrine disorders and therapeutic interventions. The manuscript management system is completely online and includes a very quick and fair peer-review system. Visit http://www.dovepress.com/testimonials.php to read real quotes from published authors. 